\title{
MicroRNA-873 Promotes Cell Proliferation, Migration, and Invasion by Directly Targeting TSLC1 in Hepatocellular Carcinoma
}

\author{
Guoyong Han ${ }^{\mathrm{a}}$ Long Zhang ${ }^{\mathrm{a}}$ Xuhao $\mathrm{Ni}^{\mathrm{a}}$ Zhiqiang Chen ${ }^{\mathrm{a}}$ Xiongxiong Pan ${ }^{\mathrm{b}}$ \\ Qin Zhu ${ }^{\mathrm{a}}$ Shu Lic Jindao Wu $\mathrm{Winl}^{\mathrm{a}}$ Xinanga,d Xuehao Wang ${ }^{\mathrm{a}}$ \\ aKey Laboratory of Living Donor Liver Transplantation, National Health and Family Planning \\ Commission, Department of Liver Surgery, The First Affiliated Hospital of Nanjing Medical University, \\ Nanjing, 'Department of Anesthesiology, The First Affiliated Hospital of Nanjing Medical University, \\ Nanjing, 'Department of General Surgery, Jiujiang University Hospital, Jiujiang, dDepartment of \\ Hepatobiliary Surgery, The Affiliated Drum Tower Hospital of Nanjing University Medical School, \\ Nanjing, P.R. China
}

\section{Key Words}

Mirna-873 • Hepatocellular carcinoma • TSLC1 • PI3K/AKT/mTOR

\begin{abstract}
Background/Aims: Hepatocellular carcinoma (HCC) is the most common type of primary liver cancer and has the third highest mortality rate among all cancers. MicroRNAs are a class of endogenous, single-stranded short noncoding RNAs. The purpose of this study was to study the role of microRNA-873 in HCC. Methods: The expression of miRNA-873 and tumor suppressor in lung cancer 1 (TSLC1) in HCC tissues and cell lines was detected by real-time quantitative RT-PCR (RT-qPCR) or western blot. A CCK-8 assay was used to examine cell proliferation; flow cytometry was used to assess the cell cycle; the Transwell migration assay was used to test for metastasis. Luciferase assays were performed to assess whether TSLC1 was a novel target of miRNA-873. Results: We showed that miRNA-873 was upregulated in HCC tissues and cell lines compared with the normal control. Knockdown of miRNA-873 inhibited the growth and metastasis of HepG2 and accelerated G1 phase arrest, while overexpression of miRNA-873 had the opposite effect. The dual-luciferase reporter assays revealed that TSLC1 was a novel target of miRNA-873. Further study showed that TSLC1 was decreased in HCC tissues and cell lines. There was a negative correlation between the expression levels of TSLC1 and miRNA-873. The effect of miRNA-873 overexpression was neutralized by TSLC1. We also found that miRNA-873 activated the PI3K/AKT/mTOR signaling pathway and promoted HCC. Conclusions: Our data demonstrated that miRNA-873 promoted HCC progression by targeting TSLC1 and provided a new target for the therapy of HCC.

G. Han, L. Zhang and X. Ni are co-first authors.

Xuehao Wang, Xinli Huang and Jindao Wu

Department of Liver Surgery, The First Affiliated Hospital of Nanjing Medical University, 300 GuangZhou Road, Nanjing, Jiangsu Province (People's Republic of China)

Tel. +86 025 83718836-6476, Fax +86 025 83672106, E-Mail wangxh@njmu.edu.cn
\end{abstract}




\section{Cellular Physiology Cell Physiol Biochem 2018;46:2261-2270

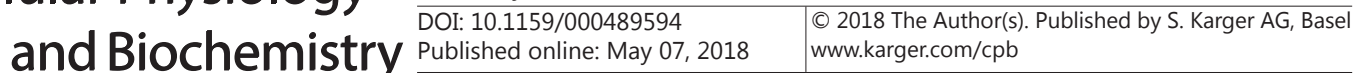 \\ Han et al.: MicroRNA-873 Promotes Progression of Hepatocellular Carcinoma}

\section{Introduction}

Hepatocellular carcinoma (HCC) is one of the most common cancers in the world and the third most common tumor-related cause of death [1,2]. Although surgical resection, liver transplantation and interventional therapy have achieved great progress in recent years, ineffective diagnosis, as well as progression and migration, leads to dissatisfactory overall survival. Therefore, it is urgent to study the mechanism of HCC progression and provide an effective therapeutic strategy.

MicroRNAs (miRNAs) are a new class of small non-coding RNAs that regulate gene expression by translational repression or degradation by targeting complementary sequences of mRNAs in the 3'-untranslated region (3'-UTR) [3, 4]. It has been reported in multiple studies that miRNAs are involved in the biology of many tumors, playing roles in cell proliferation, differentiation, motility, apoptosis, angiogenesis and metastasis [5-9]. In recent years, many miRNAs have been recognized to promote progression of HCC through enhancing oncogene expression or inhibiting tumor suppressor genes [10-12]. miRNA-873 has been reported to be frequently and aberrantly expressed in many cancers, such as glioblastoma [13], lung adenocarcinoma [14], breast cancer [15] and ovarian cancer [16]. miRNA-873 inhibits ER (estrogen receptor)- $\alpha$ activity and cell growth by targeting CDK3, mediating tamoxifen resistance [15]. miRNA-873 is downregulated in glioblastoma, inhibiting cell proliferation, migration and invasion and inducing apoptosis through suppressing the expression of IGF2BP1[13]. However, it is upregulated in lung adenocarcinoma, acting as an oncogene. The role of miRNA-873 in HCC has not yet been investigated. In this study, we sought to investigate the role miRNA-873 in HCC progression, aiming to provide a deeper understanding of the causal mechanisms of cancer cell invasion and metastasis.

\section{Materials and Methods}

Tissues and cell lines

Tumor tissue samples were taken from patients with primary HCC. All the patients underwent curative resection at the First Affiliated Hospital of Nanjing Medical University (Nanjing, China). Written informed consent was obtained from each patient before surgery. This study was approved by the Ethics Committee of the First Affiliated Hospital of Nanjing Medical University.

Cell lines used in this study included four HCC cell lines (Hep3B, HepG2, SMMC-7721, and Huh-7) and one human normal hepatic cell line (L02). All the cell lines were purchased from the Chinese Academy of Sciences Cell Bank (Shanghai, China). All cells were cultured in Dulbecco's modified Eagle's medium (DMEM, Invitrogen, Carlsbad, CA, USA) supplemented with 10\% (v/v) fetal bovine serum (HyClone, South Logan, UT, USA), 100 units $/ \mathrm{mL}$ penicillin and $100 \mu \mathrm{g} / \mathrm{mL}$ streptomycin, incubated at $37^{\circ} \mathrm{C}$ in $5 \% \mathrm{CO}_{2}$.

\section{RNA isolation and real-time quantitative RT-PCR}

Total RNA was extracted using TRIzol Reagent (Takara, Dalian, China) according to the manufacturer's protocol. The quality and concentration were detected using a Nanodrop 2000 (NanoDrop Technologies, Massachusetts, USA). RT-qPCR was performed with SYBR Premix ExTaq (TaKaRa) with the ABI Prism 7900 HT (Applied Biosystems, Foster City, CA, USA). U6 was used as internal control for mRNA quantification. The relative expression ratio of miR-873 and TSLC1 in each tissue and cell line was calculated using the $2^{-\triangle \Delta C T}$ method.

\section{Western blotting}

Cells and tissue specimens were lysed using RIPA buffer (Sigma-Aldrich, St. Louis, MO, USA). Protein samples $(20 \mu \mathrm{g})$ were separated using $10 \%$ sodium dodecyl sulfate-polyacrylamide gel electrophoresis and then transferred to a polyvinylidene difluoride membrane. The membranes were blocked with $5 \%$ bovine serum albumin in Tris-buffered saline containing 0.1\% TWEEN 20 at room temperature for $2 \mathrm{~h}$ and incubated in primary antibody overnight at $4{ }^{\circ} \mathrm{C}$. After being washed with TBST three times for 15 min each, the membrane was incubated with the appropriate horseradish peroxidase-conjugated secondary 


\section{Cellular Physiology Cell Physiol Biochem 2018;46:2261-2270 \begin{tabular}{l|l} 
DOI: 10.1159/000489594 & $\begin{array}{l}\text { O 2018 The Author(s). Published by S. Karger AG, Basel } \\
\text { www.karger.com/cpb }\end{array}$
\end{tabular} \\ Han et al.: MicroRNA-873 Promotes Progression of Hepatocellular Carcinoma}

antibody for $2 \mathrm{~h}$ at room temperature. After the membrane was washed again with TBST as described previously, proteins were visualized with an enhanced chemiluminescence detection kit according to the manufacturer's recommendations (Beyotime, Nantong, China). Protein levels were calculated relative to GAPDH.

\section{Cell transfection}

Lipofectamine 3000 (Invitrogen, Carlsbad, CA, USA) was used to perform miRNA transfection according to the manufacturer's protocol. Cells were plated in six-well plates at a density of $1.5 \times 10^{5}$ cells per well the day before transfection. When the cells were in the log phase, an miRNA-873 inhibitor or mimic (Genepharma, Shanghai, China) was added to the well. The medium was replaced after a 6-h incubation period. Cells were harvested 48-72 h later for analysis. For the transfection of TSLC1, lentiviruses overexpressing TSLC1 (lv-TSLC1) and corresponding control lentiviruses (lv-green fluorescent protein) were constructed by Genepharma (Shanghai, China). Cells were plated in six-well plates at a density of $2 \times 10^{5}$ cells per well. When the density reached $30 \%-40 \%$ after incubation, lentiviruses were transfected into HCC cells with a multiplicity of infection of 10 . Seventy-two hours after infection, cells were selected for 2 weeks using puromycin $(10 \mu \mathrm{g} / \mathrm{mL})$. The selected cell lines were analyzed for TSLC1 expression and prepared for subsequent experiments.

\section{Luciferase assay}

MiRNA targets were predicted using the algorithm TargetScan. Cells $\left(5 \times 10^{4}\right)$ were seeded in 24-well plates and cultured for $24 \mathrm{~h}$. The TSLC1 reporter luciferase plasmid (100 ng), pGL3-TSLC1, pGL3-TSLC1mut or control luciferase plasmid, and 5 ng pRL-TK Renilla plasmid (Promega,Madison, WI, USA) were transfected into the cells using Lipofectamine 3000 according to the manufacturer's instructions. Luciferase and Renilla signals were measured $48 \mathrm{~h}$ after transfection using a Dual-Luciferase Reporter Assay Kit (Promega) according to the manufacturer's protocol.

\section{CCK-8 assay}

The Cell Counting Kit-8 (CCK-8, Dojindo, Kumamoto, Japan) was used in this study. Cells were plated in 96-well plates at a density of 3000 cells/well. Ten microliters of CCK- 8 was added to each well at several time points $(0,24,48,72$ and $96 \mathrm{~h})$. The cells were incubated at $37^{\circ} \mathrm{C}$ for $\underline{2}$ hours. The absorbance at $450 \mathrm{~nm}$ was measured to generate cell growth curves.

\section{Transwell assay}

The migration and invasion assay was performed using Transwell chambers (Corning Incorporated, Corning, NY, USA). For migration, $4 \times 10^{4}$ cells were resuspended in serum-free medium and seeded into a Transwell insert supplemented with DMEM containing 5\% serum. After 24-48 h incubation, the cells on the bottom side of the membrane were fixed with $95 \%$ alcohol and stained with crystal violet for 20 min at room temperature. Then, the number of cells on the lower side of the filter was counted under a microscope. Each experiment was performed in triplicate. For invasion, Matrigel was applied to the upper surface before the cells were added. The other steps were the same as for the migration assay.

\section{Flow cytometry analysis}

A Cell Cycle Detection Kit (Vazyme Biotech, Nanjing, China) was used. Cells were plated in a six-well plate and cultured for $48 \mathrm{~h}$. Then, the cells were collected and fixed with $70 \%$ ethanol at $4{ }^{\circ} \mathrm{C}$ for more than $6 \mathrm{~h}$ and then washed with phosphate-buffered saline (PBS) twice. The staining was carried out with $50 \mu \mathrm{g} /$ $\mathrm{mL}$ propidium iodide in PBS-Triton X-100 for $30 \mathrm{~min}$ at room temperature in the absence of light. Finally, cells were analyzed on a FACSCalibur flow cytometer equipped with CellQuest software (BD Biosciences, New York, NY, USA).

\section{Statistical analysis}

SPSS 18.0 (SPSS, Chicago, IL, USA) or Prism 5.0 (GraphPad Software, La Jolla, CA, USA) software was used for statistical analyses. The results are presented as the mean \pm standard error of the mean. Quantitative and categorical data were subjected to the two-tailed Student's t-test and the $\chi^{2}$ test, respectively. $P<0.05$ was considered statistically significant.

\section{KARGER}




\section{Cellular Physiology Cell Physiol Biochem 2018;46:2261-2270 and Biochemistry Published onIne: Vay 07, $2018 \quad \begin{aligned} & \text { DOI: 10.1159/000489594 } 2018 \text { The Author(s). Published by S. Karger AG, Basel } \\ & \text { www.karger.com/cpb }\end{aligned}$ \\ Han et al.: MicroRNA-873 Promotes Progression of Hepatocellular Carcinoma}

\section{Results}

\section{miRNA-873 is upregulated in HCC and correlates with clinical characteristics}

We first detected miRNA-873 expression by qRT-PCR. As shown in Fig. 1A, miRNA-873 was significantly upregulated in three HCC cell lines (Hep3B, HepG2, and SMCC-7721) compared with a normal liver cell line (L02). Furthermore, we detected miRNA-873 expression in HCC tissues. A large increase in miRNA-873 was observed in HCC tissues compared with adjacent non-cancerous tissues (Fig. 1B). These data demonstrate that miRNA-873 expression was elevated in HCC. We further analyzed the correlation between miRNA-873 expression and clinical parameters in 70 HCC patients. As shown in Table 1, the expression of miRNA-873 was correlated with alpha fetal protein $(P=0.0209)$, tumor size $(P=0.0052)$, vascular invasion $(P=0.0025)$ and Edmondson grade $(P=0.0011)$. These data indicate that miR-873 might promote HCC development.

\section{miRNA-873 pro- \\ moted prolifera- \\ tion, migration and \\ invasion by $\mathrm{HCC}$ \\ cells \\ To explore the} biological function of miRNA-873 in HCC, we transfected an miRNA-873 inhibitor or mimic into HepG2 and Huh7 cells, respectively. Real-time PCR showed that miRNA-873 expression was significantly changed after transfection (Fig. 2A and $3 \mathrm{~A})$. HepG2 transfected with miRNA-873 inhibitor showed a decreased cell growth rate and an impaired capacity for migration and invasion. The cell cycle was arrested at G1 (Fig. 2B-D). Huh7 cells transfected with miRNA-873 mimic showed an increased growth rate and enhanced migration and invasion ability. Cell cycle analysis showed that miRNA-873 accelerated the $S$ phase (Fig. 3B-D). These data demonstrate that miRNA-873 promotes HCC cell proliferation, migration and invasion.

TSLC1 is a direct target of miRNA-873 in HCC cells

We found that TSLC1, whose 3'-UTR was complementary to miRNA-873, could be a potential target of that miRNA (Fig. 4A). TSLC1 has been

Table 1. Clinical characteristics of HCC patients and correlation with miRNA-873 levels. ${ }^{*} \mathrm{P}<0.05$, ${ }^{* * *} \mathrm{P}<0.01$

\begin{tabular}{lcccc}
\hline Variable & Patients (n=70) & miR-873 high & miR-873 low & P value \\
\hline Age (y) & $\geq 55$ & 20 & 19 & 0.5715 \\
& $<55$ & 18 & 13 & \\
Gender & Male & 30 & 28 & 0.3442 \\
& Female & 8 & 4 & \\
AFP (ng/mL) & $\geq 400$ & 27 & 14 & $0.0209^{*}$ \\
& $<400$ & 11 & 18 & \\
Tumor number & Single & 24 & 17 & 0.3959 \\
& Multiple & 14 & 15 & \\
Tumor size (cm) & $\geq 5$ & 28 & 13 & $0.0052^{* *}$ \\
& $<5$ & 10 & 19 & \\
Vascular invasion & Yes & 19 & 5 & $0.0025^{* *}$ \\
& No & 19 & 27 & \\
Edmondson grade & I-II & 15 & 25 & $0.0011^{* *}$ \\
& III-IV & 23 & 7 & \\
\hline
\end{tabular}




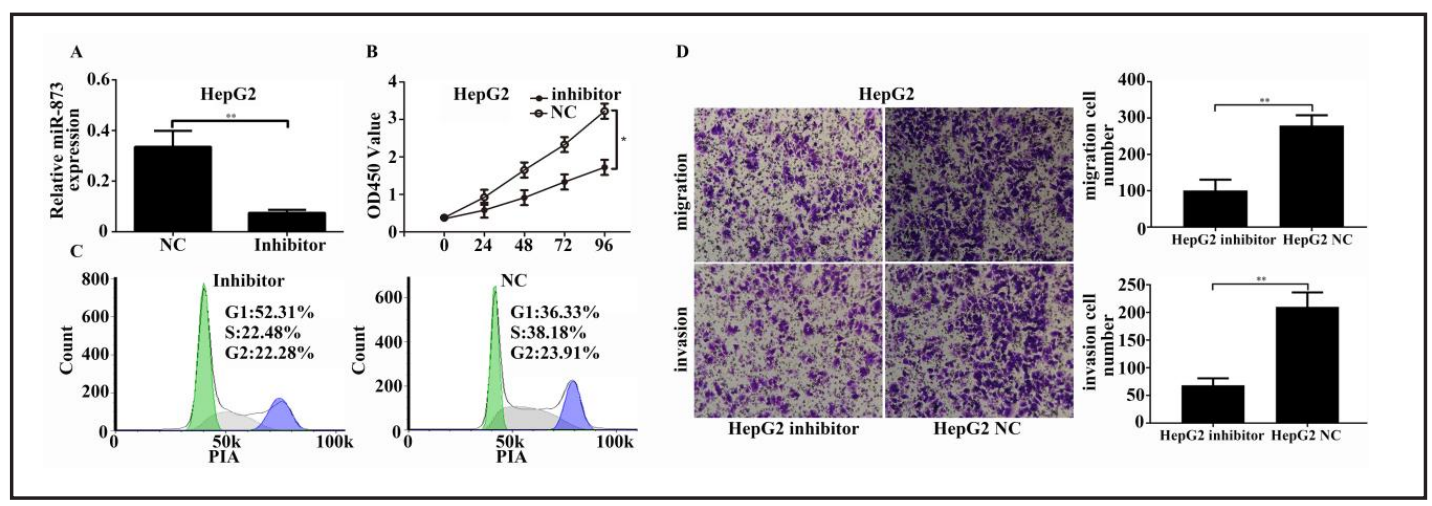

Fig. 2. The effects of miRNA-873 inhibition on cell growth, migration, and invasion by the HCC cell line HepG2. (A) Real-time PCR was used to measure miRNA-873 expression levels after transfection with an miRNA-873 inhibitor or negative control vector. (B) A CCK-8 assay was used to show the effect of miRNA-873 inhibition on the HepG2 cell proliferation rate. (C) Flow cytometry was used to show the effect of miRNA-873 inhibition on the HepG2 cell cycle. (D) Transwell assay was used to show the effect of miRNA-873 inhibition on HepG2 migration and invasion. ${ }^{*} \mathrm{P}<0.05,{ }^{* *} \mathrm{P}<0.01$.

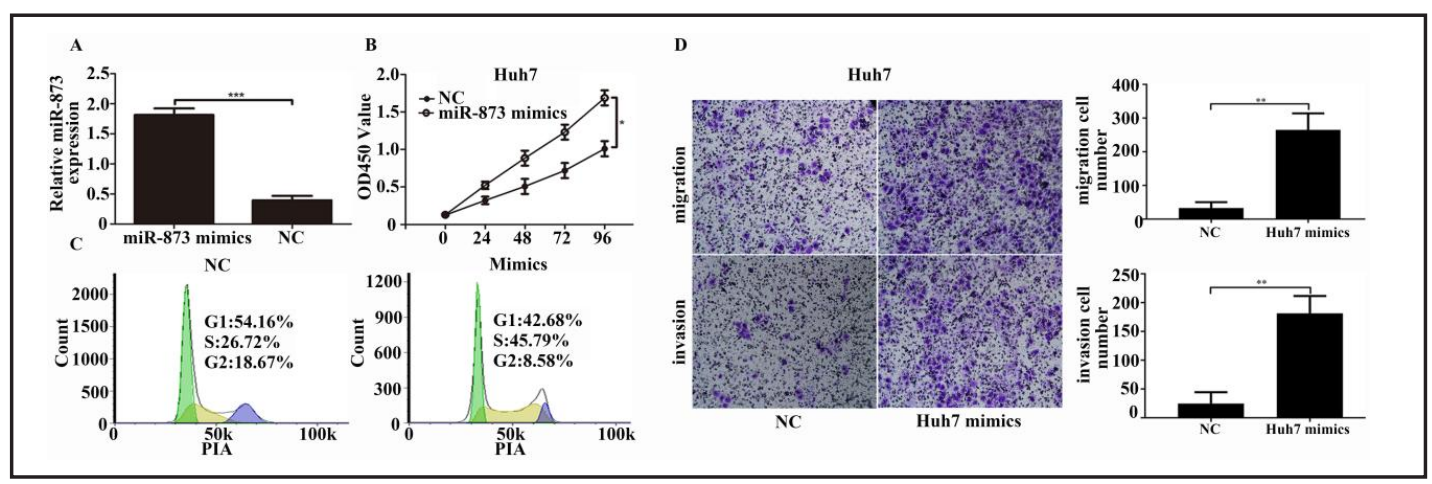

Fig. 3. The effects of miRNA-873 overexpression on the cell growth, migration and invasiveness of Huh7 cells. (A) Real-time PCR was used to measure miRNA-873 expression levels after transfection with a miRNA-873 mimic or negative control vector. (B) A CCK-8 assay was used to show the effect of miRNA-873 overexpression on the Huh7 cell proliferation rate. (C) Flow cytometry was used to show the effect of miRNA-873 overexpression on the Huh7 cell cycle. (D) A Transwell assay was used to show the effect of miRNA-873 overexpression on Huh7 migration and invasion. ${ }^{*} \mathrm{P}<0.05$, ${ }^{* *} \mathrm{P}<0.01$.

reported as a tumor suppressor gene in many tumors, including HCC $[17,18]$. We detected the expression of TSLC1 in HCC and found that TSLC1 was significantly decreased in HCC cell lines and tissues (Fig. 4B). Furthermore, Spearman's correlation analysis showed that TSLC1 mRNA levels were inversely correlated with miRNA-873 levels (Fig. 4C). To verify their connection, we conducted dual luciferase reporter experiments. The result showed that overexpressed miRNA-873 could significantly suppress the luciferase activity of the wild 3'-UTR of TSLC1 but not the mutant 3'-UTR of TSLC1 (Fig. 4D). These data demonstrate that miRNA-873 can directly target TSLC1 and suppress its expression.

\section{TSLC1 rescued the effect of miRNA-873-mediated HCC progression}

To determine whether TSLC1 is involved in miRNA-873-regulated HCC cell proliferation, migration, and invasion, we used a lentiviral vector overexpressing TSLC1. Huh7 cells transfected with miRNA-873 mimics were infected with lv-TSLC1. We found that increased TSLC1 could rescue the effect of miRNA-873 mimics on proliferation, migration and invasion in Huh7 cells (Fig. 5A, B, C). These results indicate that miRNA-873 exerted its accelerative effects in HCC cells by repressing TSLC1. 


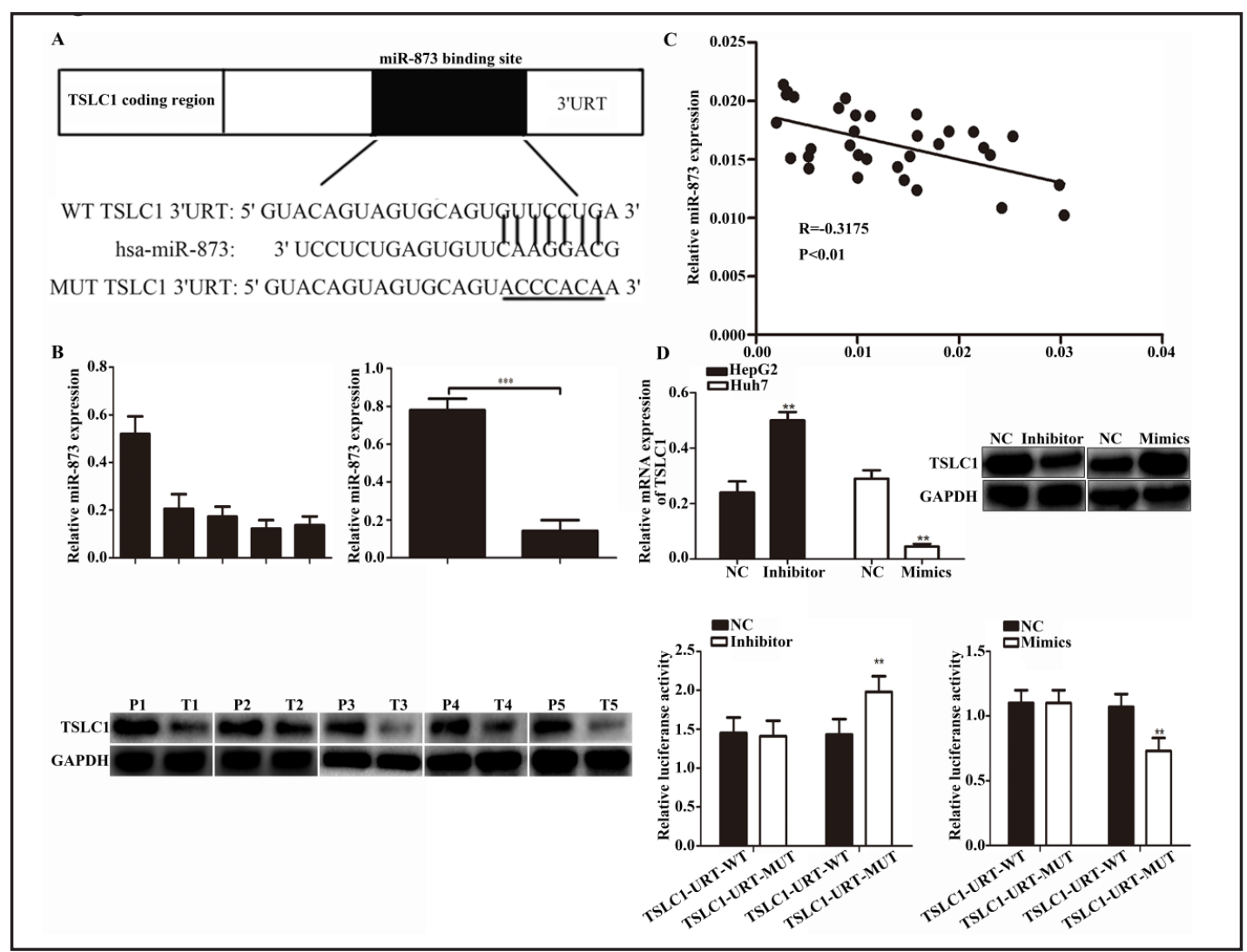

Fig. 4. TSLC1 is a direct target of miRNA-873. (A) The predicted interactions between miRNA-873 and its target sites in the TSLC1 3'-untranslated region (UTR) are shown. (B) miRNA-873 was overexpressed in HCC as shown by real-time PCR and western blotting. (C) The expression levels of TSLC1 and miRNA-873 in HCC are inversely correlated. (D) miRNA-873 overexpression or downregulation inhibited or enhanced, respectively, the luciferase activity of the reporter gene with the wild-type construct but not the mutant TSLC1 3'-UTR construct. ${ }^{* *} \mathrm{P}<0.01$.

miRNA-873 might promote HCC progression by activating the PI3K/AKT/mTOR

We used western blotting to further explore the potential signaling pathway of miRNA873-induced HCC progression. The results showed that overexpression of miR-873 could significantly induce PI3K, AKT and mTOR phosphorylation. Conversely, inhibition of miRNA-873 effectively downregulated phosphorylated PI3K, AKT and phosphorylated mTOR (Fig. 6). These data demonstrate that miRNA-873 promoted HCC proliferation, migration, and invasion by activating the $P I 3 K / A K T / m T O R$ signaling pathway.

\section{Discussion}

HCC is one of the most common cancers in the world, especially in Asia. Because of the poor overall survival associated with it, new therapies are urgently needed. MicroRNAs have been found to play a dual role in the progression of tumors, including HCC: some act as oncogenes, and some as tumor suppressor genes [19-21]. For example, miR-155 is upregulated in HCC, promotes cancer cell invasion, and is a predictor of poor survival of hepatocellular carcinoma [22]. Additionally, hepatitis C virus-induced upregulation of miR155 promotes hepatocarcinogenesis by activating Wnt signaling [23]. In HCC cell lines, microRNA-133a suppresses proliferation, colony formation, migration and invasion and induces cell cycle arrest at the G0/G1 stage, as well as cell apoptosis, indicating a tumor suppression function [24]. miR-34a acts as a tie molecule between p53 and SIRT1 and exerts a tumor suppression effect in HCC by promoting cell apoptosis [25].

\section{KARGER}




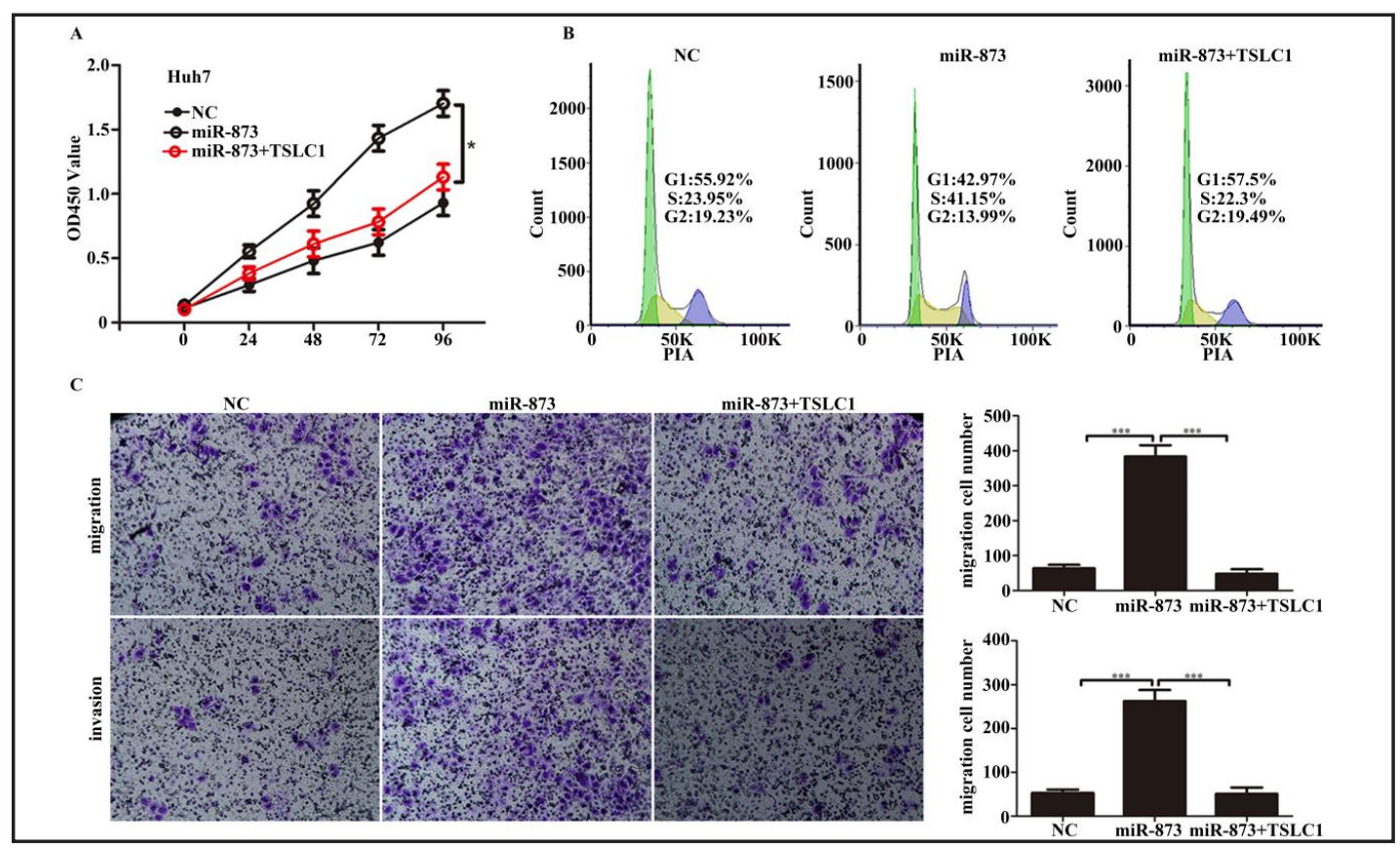

Fig. 5. The effect of TSLC1 overexpression on miRNA-873-mediated HCC progression. (A) Real-time PCR and western blot analyses were used to measure TSLC1 expression after transfection with miRNA-873 mimic and lv-TSLC1 vectors. (B) Cell proliferation and the cell cycle were assessed in Huh7 cells transfected with miRNA-873 with and without TSLC1 overexpression. (C) Cell migration and invasion were determined in Huh7 cells transfected with miRNA-873 with and without TSLC1 overexpression. ${ }^{*} \mathrm{P}<0.05,{ }^{* * *} \mathrm{P}<0.01$.

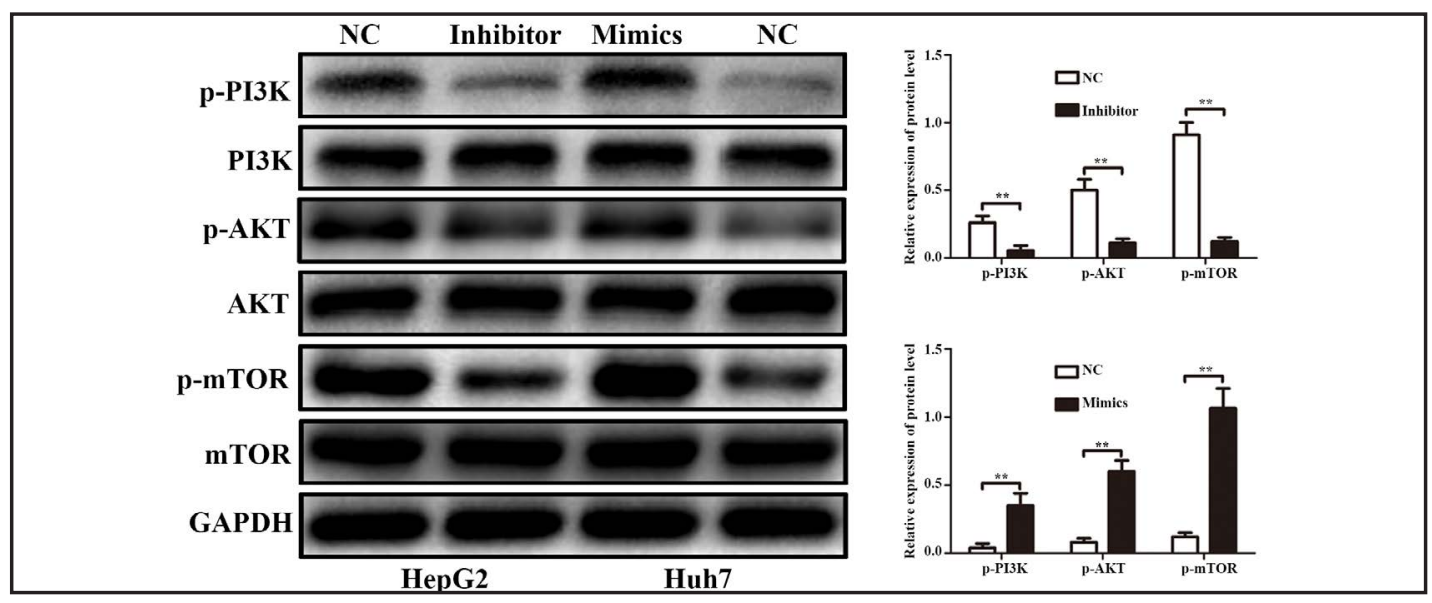

Fig. 6. miR-873 activates the PI3K/AKT/mTOR signaling pathway. Western blot analysis of PI3K/AKT/ mTOR and their activation in HepG2 cells transfected with a negative control, miR-873 inhibitor or miR-873 mimic vector.

Recent studies have shown that miRNA-873 is frequently altered in multiple tumor types and is associated with the pathogenesis and progression of cancer. However, it plays different roles in different tumors. miRNA-873 promotes the proliferation and migration of tumor cells by repressing Src in lung adenocarcinoma [14] but acts as a tumor suppressor in glioblastoma [13] and ovarian cancer [16]. However, little is known about the role of miRNA-873 in HCC. In the present study, we found that miRNA-873 was significantly increased in HCC tissues and tumor cell lines. miRNA-873 significantly promoted HCC cell proliferation, migration and invasion. 
TSLC1 has been reported as a tumor suppressor gene that is involved in multiple cellular processes such as cell proliferation, differentiation, migration, and invasion [26, 27]. Tsujiuchi et al. reported that in HCC induced by N-nitrosodiethylamine, the $5^{\prime}$ upstream region of TSLC1 was methylated and its expression was reduced [18]. Additionally, some researchers reported that restoration of TSLC1 could effectively inhibit HCC growth [28]. In the present study, we were the first to identify TSLC1 as a direct target of miRNA-873 in HCC cells. TSLC1 expression was decreased in HCC tissues and cell lines. TSLC1 overexpression rescued the effect of miRNA-873-mediated HCC cell proliferation, migration, and invasion. These results suggest that miRNA-873 exerts a tumor-accelerative role in HCC at least partly through repressing TSLC1 expression, and because of the significant decrease of TSLC1 in HCC, it may be an important prognostic indicator for HCC patients. These results together suggest that miRNA-873 could be used as a potential therapeutic target for HCC.

A number of studies have demonstrated that the $P I 3 K / A K T / m T O R$ signaling pathway is excessively activated in tumors, induces tumorigenesis and promotes tumor progression $[29,30]$. Under physiological conditions, PI3K/AKT signaling pathway is one of the important signal transduction pathways and plays a key role in inhibiting apoptosis and promoting proliferation by affecting the activation state of various downstream effector molecules [31]. Many factors could cause aberrant phosphorylation of PI3K/AKT/mTOR, such as interleukin-6[32]. We further tested whether this mechanism is involved in the promotion of HCC by miRNA-873. Our data show that miRNA-873 can significantly phosphorylate PI3K/ $A K T / m T O R$ and that inhibition of miRNA-873 can inactivate these two factors.

\section{Conclusion}

In summary, our study was the first to show that miRNA-873 exerts a tumor-accelerative function in HCC by regulating cell growth and metastasis. Additionally, miRNA-873 promoted HCC progression by repressing TSLC1. The potential mechanism might be the excessive phosphorylation of PI3K/AKT/mTOR as an effect of miRNA-873. These results suggest that miRNA-873 might be employed as a novel and effective therapeutic target for HCC in the future.

\section{Acknowledgements}

This work was supported by the Six Talent Peaks Project of Jiangsu Province (Grant No. N02014-WSW-005) and the National Natural Science Foundation of China (Grant No. 81300363).

\section{Disclosure Statement}

The authors declare no Disclosure Statement.

\section{References}

1 Venook AP, Papandreou C, Furuse J, de Guevara LL: The incidence and epidemiology of hepatocellular carcinoma: a global and regional perspective. Oncologist 2010;15 Suppl 4:5-13.

2 Chen W, Zheng R, Baade PD, Zhang S, Zeng H, Bray F, Jemal A, Yu XQ He J: Cancer statistics in China, 2015. CA Cancer J Clin 2016;66:115-132.

3 Kim VN, Nam JW: Genomics of microRNA. Trends Genet 2006;22:165-173.

4 Olena AF, Patton JG: Genomic organization of microRNAs. J Cell Physiol 2010;222:540-545.

5 Wiemer EA: The role of microRNAs in cancer: no small matter. Eur J Cancer 2007;43:1529-1544. 


\section{Cellular Physiology Cell Physiol Biochem 2018;46:2261-2270 \begin{tabular}{l|l} 
DOI: 10.1159/000489594 & $\begin{array}{l}\text { O 2018 The Author(s). Published by S. Karger AG, Basel } \\
\text { www.karger.com/cpb }\end{array}$
\end{tabular} \\ Han et al.: MicroRNA-873 Promotes Progression of Hepatocellular Carcinoma}

6 Wienholds E, Plasterk RH: MicroRNA function in animal development. FEBS Lett 2005;579:5911-5922.

7 Sun JJ, Chen GY, Xie ZT: MicroRNA-361-5p Inhibits Cancer Cell Growth by Targeting CXCR6 in Hepatocellular Carcinoma. Cell Physiol Biochem 2016;38:777-785.

-8 Zhu H, Zhou X, Ma C, Chang H, Li H, Liu F, Lu J: Low Expression of miR-448 Induces EMT and Promotes Invasion by Regulating ROCK2 in Hepatocellular Carcinoma. Cell Physiol Biochem 2015;36:487-498.

-9 Gao F, Sun X, Wang L, Tang S, Yan C: Downregulation of MicroRNA-145 Caused by Hepatitis B Virus X Protein Promotes Expression of CUL5 and Contributes to Pathogenesis of Hepatitis B Virus-Associated Hepatocellular Carcinoma. Cell Physiol Biochem 2015;37:1547-1559.

10 Mao B, Wang G: MicroRNAs involved with hepatocellular carcinoma (Review). Oncol Rep 2015;34:28112820.

11 Giordano S, Columbano A: MicroRNAs: new tools for diagnosis, prognosis, and therapy in hepatocellular carcinoma? Hepatology 2013;57:840-847.

12 Shi L, Wu L, Chen Z, Yang J, Chen X, Yu F, Zheng F, Lin X: MiR-141 Activates Nrf2-Dependent Antioxidant Pathway via Down-Regulating the Expression of Keap1 Conferring the Resistance of Hepatocellular Carcinoma Cells to 5-Fluorouracil. Cell Physiol Biochem 2015;35:2333-2348.

-13 Wang RJ, Li JW, Bao BH, Wu HC, Du ZH, Su JL, Zhang MH, Liang HQ: MicroRNA-873 (miRNA-873) inhibits glioblastoma tumorigenesis and metastasis by suppressing the expression of IGF2BP1. J Biol Chem 2015;290:8938-8948.

14 Gao Y, Xue Q, Wang D, Du M, Zhang Y, Gao S: miR-873 induces lung adenocarcinoma cell proliferation and migration by targeting SRCIN1 Am J Transl Res 2015;7:2519-2526.

15 Cui J, Yang Y, Li H, Leng Y, Qian K, Huang Q, Zhang C, Lu Z, Chen J, Sun T, Wu R, Sun Y, Song H, Wei X, Jing P, Yang X, Zhang C: MiR-873 regulates ERalpha transcriptional activity and tamoxifen resistance via targeting CDK3 in breast cancer cells. Oncogene 2015;34:3895-3907.

-16 Wu DD, Li XS, Meng XN, Yan J, Zong ZH: MicroRNA-873 mediates multidrug resistance in ovarian cancer cells by targeting ABCB1. Tumour Biol 2016;37:10499-10506.

17 Kuramochi M, Fukuhara H, Nobukuni T, Kanbe T, Maruyama T, Ghosh HP, Pletcher M, Isomura M, Onizuka M, Kitamura T, Sekiya T, Reeves RH, Murakami Y: TSLC1 is a tumor-suppressor gene in human non-smallcell lung cancer. Nat Genet 2001;27:427-430.

18 Tsujiuchi T, Sugata E, Masaoka T, Onishi M, Fujii H, Shimizu K, Honoki K: Expression and DNA methylation patterns of Tslc1 and Dal-1 genes in hepatocellular carcinomas induced by N-nitrosodiethylamine in rats. Cancer Sci 2007;98:943-948.

19 Liu Y, Chai Y, Zhang J, Tang J: A Function Variant at miR-501 Alters Susceptibility to Hepatocellular Carcinoma in a Chinese Han Population. Cell Physiol Biochem 2016;38:2500-2508.

20 Cui L, Hu Y, Bai B, Zhang S: Serum miR-335 Level is Associated with the Treatment Response to TransArterial Chemoembolization and Prognosis in Patients with Hepatocellular Carcinoma. Cell Physiol Biochem 2015;37:276-283.

21 Zheng Y, Chen H, Yin M, Ye X, Chen G, Zhou X, Yin L, Zhang C, Ding B: MiR-376a and histone deacetylation 9 form a regulatory circuitry in hepatocellular carcinoma. Cell Physiol Biochem 2015;35:729-739.

-22 Han ZB, Chen HY, Fan JW, Wu JY, Tang HM, Peng ZH: Up-regulation of microRNA-155 promotes cancer cell invasion and predicts poor survival of hepatocellular carcinoma following liver transplantation. J Cancer Res Clin Oncol 2012;138:153-161.

-23 Yan XL, Jia YL, Chen L, Zeng Q, Zhou JN, Fu CJ, Chen HX, Yuan HF, Li ZW, Shi L, Xu YC, Wang JX, Zhang XM, He LJ, Zhai C, Yue W, Pei XT: Hepatocellular carcinoma-associated mesenchymal stem cells promote hepatocarcinoma progression: role of the S100A4-miR155-SOCS1-MMP9 axis. Hepatology 2013;57:22742286.

24 Zhang W, Liu K, Liu S, Ji B, Wang Y, Liu Y: MicroRNA-133a functions as a tumor suppressor by targeting IGF1R in hepatocellular carcinoma. Tumour Biol 2015;36:9779-9788.

25 Lou G, Liu Y, Wu S, Xue J, Yang F, Fu H, Zheng M, Chen Z: The p53/miR-34a/SIRT1 Positive Feedback Loop in Quercetin-Induced Apoptosis. Cell Physiol Biochem 2015;35:2192-2202.

26 Chen K, Wang G, Peng L, Liu S, Fu X, Zhou Y, Yu H, Li A, Li J, Zhang S, Bai Y, Zhang Y: CADM1/TSLC1 inactivation by promoter hypermethylation is a frequent event in colorectal carcinogenesis and correlates with late stages of the disease. Int J Cancer 2011;128:266-273. 


\section{Cellular Physiology Cell Physiol Biochem 2018;46:2261-2270

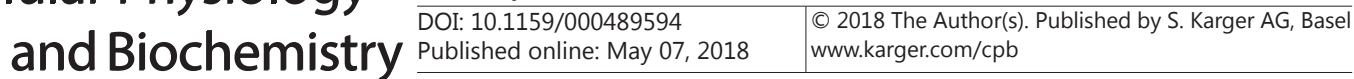 \\ Han et al.: MicroRNA-873 Promotes Progression of Hepatocellular Carcinoma}

-27 Liu D, Feng X, Wu X, Li Z, Wang W, Tao Y, Xia Y: Tumor suppressor in lung cancer 1 (TSLC1), a novel tumor suppressor gene, is implicated in the regulation of proliferation, invasion, cell cycle, apoptosis, and tumorigenicity in cutaneous squamous cell carcinoma. Tumour Biol 2013;34:3773-3783.

28 He G, Lei W, Wang S, Xiao R, Guo K, Xia Y, Zhou X, Zhang K, Liu X, Wang Y: Overexpression of tumor suppressor TSLC1 by a survivin-regulated oncolytic adenovirus significantly inhibits hepatocellular carcinoma growth. J Cancer Res Clin Oncol 2012;138:657-670.

-29 Chen Y, Zhou X, Qiao J, Bao A: MiR-142-3p Overexpression Increases Chemo-Sensitivity of NSCLC by Inhibiting HMGB1-Mediated Autophagy. Cell Physiol Biochem 2017;41:1370-1382.

-30 Wolin EM: PI3K/Akt/mTOR pathway inhibitors in the therapy of pancreatic neuroendocrine tumors. Cancer Lett 2013;335:1-8.

-31 Martelli AM, Chiarini F, Evangelisti C, Grimaldi C, Ognibene A, Manzoli L, Billi AM, McCubrey JA: The phosphatidylinositol 3-kinase/AKT/mammalian target of rapamycin signaling network and the control of normal myelopoiesis. Histol Histopathol 2010;25:669-680.

-32 Chen H, Zhou L, Wu X, Li R, Wen J, Sha J, Wen X: The PI3K/AKT pathway in the pathogenesis of prostate cancer. Front Biosci (Landmark Ed) 2016;21:1084-1091 